\title{
RUBRICS FOR ACCREDITATION AND OUTCOMES ASSESSMENT in Engineering Capstone Projects
}

\author{
William Bishop \\ Department of Electrical and \\ Computer Engineering \\ University of Waterloo \\ Waterloo, ON, N2L 3G1 \\ wdbishop@uwaterloo.ca
}

\author{
Oscar Nespoli \\ Department of Mechanical and \\ Mechatronics Engineering \\ University of Waterloo \\ Waterloo, ON, N2L 3G1 \\ oscar@uwaterloo.ca
}

Wayne Parker

Department of Civil and

Environmental Engineering

University of Waterloo

Waterloo, ON, N2L 3G1

wjparker@uwaterloo.ca

\begin{abstract}
Capstone projects offer an excellent opportunity to assess the attributes of engineering students in their final year of studies. For the purposes of accreditation and outcomes assessment, capstone projects can be used to establish that engineering students have obtained a suitable level of mastery of the skills necessary to be successful in their field of study. At the University of Waterloo, a committee was formed by the Faculty of Engineering to investigate, develop, and implement a common set of rubrics for the purpose of consistently assessing graduate attributes across all engineering disciplines. Faculty members from every engineering discipline were appointed to the committee. Using the collective experience of the committee members, a set of rubrics for outcomes assessment was established.
\end{abstract}

This paper examines the design of the six rubrics that the committee deemed to be equally applicable to all engineering disciplines. These rubrics assess the $C E A B$ graduate attributes of problem analysis, design, individual and team work, communication skills, and economics and project management. Each rubric subdivides the assessment of an attribute into a set of elements that are examined independently. This paper presents the rubrics, examines the elements of each $C E A B$ graduate attribute, and examines the expected level of mastery associated with each assessment level. This paper concludes with a discussion of the recent use of the rubrics in the assessment of capstone projects in the Department of Electrical and Computer Engineering.

Keywords: CEAB Accreditation, Outcomes Assessment, Engineering Capstone Projects

\section{INTRODUCTION}

In the field of engineering, outcomes-based assessment is a relatively new idea. In 1996, ABET (Accreditation Board for Engineering and Technology), the body responsible for accrediting undergraduate engineering programs in the United States of America, adopted a set of standards known as EC2000 [1]. The EC2000 standards shifted the focus of engineering accreditation from measuring what is taught (inputs) to measuring what is learned (outputs). Many reasons are often cited for this transition towards outcomes-based assessment. One of the most commonly cited reasons is that outcomes-based assessment can help facilitate a process of continual improvement.

In the Province of Ontario, undergraduate engineering programs are transitioning towards being evaluated against two different outcomes-based assessment models. The first model, adopted by the CEAB (Canadian Engineering Accreditation Board), focuses specifically on the outcomes expected of graduates of undergraduate engineering programs in Canada. The second model, adopted by the OCAV (Ontario Council of Academic Vice-Presidents), focuses on the outcomes expected of all graduates of undergraduate programs in the Province of Ontario.

\subsection{CEAB Accreditation}

Since 1965, Engineers Canada (formerly the Canadian Council of Professional Engineers) has maintained a Canadian Engineering Accreditation Board to accredit Canadian undergraduate engineering programs [2]. The purpose of accreditation is to identify engi- 
neering programs whose graduates are academically qualified to begin the process of licensure as a professional engineer in Canada.

According to Section 3.1 of the Canadian Engineering Accreditation Board report entitled "Accreditation Criteria and Procedures" [2], starting in June 2015, institutions must demonstrate that the graduates of a program possess the following set of twelve attributes:

1) A knowledge base for engineering,

2) Problem analysis,

3) Investigation,

4) Design,

5) Use of engineering tools,

6) Individual and team work,

7) Communication skills,

8) Professionalism,

9) Impact of engineering on society and the environment,

10) Ethics and equity,

11) Economics and project management, and

12) Life-long learning.

Some of the attributes examine discipline specific skills (e.g., a knowledge base for engineering, design, and the use of engineering tools) while other attributes examine higher order skills (e.g., communication skills, professionalism, and life-long learning). Given that these attributes must be obtained prior to graduation, the best time for attribute assessment is often in the final year of a student's program of study.

\subsection{UDLE Assessment}

In 2005, OCAV adopted a set of outcomes-based assessments known as UDLEs [3] that came into effect in June 2008. The UDLE assessment model focuses on skills that are generally applicable to all undergraduate programs. This model requires that graduates of a program meet the following expectations:

1) Depth and breadth of knowledge,

2) Knowledge of methodologies,

3) Application of knowledge,

4) Communication skills,

5) Awareness of limits of knowledge, and

6) Autonomy and professional capacity.

In addition to these six core expectations, institutions are encouraged to establish additional expectations for programs as a means of identifying particular areas of strength. The University of Waterloo has established the following two additional expectations to satisfy:

1) Experiential learning

2) Diversity
These additional expectations recognize the importance of the University of Waterloo's co-operative work experience program and the University of Waterloo's international reputation. By offering an expectation of experiential learning and diversity, the University of Waterloo distinguishes itself from other institutions in the province.

UDLE assessment is best performed in the final year of a student's program of study. Using feedback provided by regular assessments of graduating classes, it is possible to establish a continual improvement process to ensure that expectations are always met or exceeded.

\subsection{Capstone Projects}

In the field of engineering, a capstone project allows a final year student to work as part of a team on a engineering design project that requires the application of knowledge and skills acquired through course work. To complete capstone projects, students leverage the engineering design process to propose, design, analyze, assemble, test, and present a prototype design. Capstone projects exercise both discipline specific skills and higher order skills. As a result, capstone projects provide an excellent opportunity to assess the outcomes of an undergraduate degree program.

The core UDLEs have a direct overlap with one or more CEAB attributes so it is possible to satisfy many of the requirements of both outcomes-based assessment models using a single process. Using a carefully designed set of rubrics for capstone projects, educators can obtain assessments to satsify both outcomes-based assessment models.

\section{BACKGROUND}

At the University of Waterloo, all engineering students are expected to participate in a design project in their final year of studies. One of the goals of this design project is to satisfy the CEAB requirement for a significant design experience based on the knowledge and skills acquired through course work. Additionally, the design project provides an opportunity to assess many of the higher order skills that graduates of a university degree program are expected to possess.

A committee was formed to examine the feasibility of developing a set of rubrics for capstone project assessment to be used by the entire Faculty of Engineering. This committee consisted of faculty member representatives from each engineering discipline. In most cases, the faculty members appointed to the committee were faculty members who had previous 
experience co-ordinating capstone projects within their department.

It should be noted that rubrics play an important role in the curriculum, not only as a means of assessing student performance but also as a tool for enhancing the curriculum. While the primary goal of the committee was to develop a consistent assessment mechanism for capstone projects, the committee also hoped to achieve several secondary goals. These goals included the following:

1) Educate instructors about outcomes assessment as a pedagogical strategy,

2) Drive curriculum development by providing a framework to assess the "connectivity" of instruction throughout a program, and

3) Inform students about the key elements of of their program.

\subsection{Opportunities}

Some of the opportunities afforded by the use of a common set of rubrics are the following:

1) Capstone projects can be assessed consistently across all disciplines,

2) Assessment statistics from each department can be compared, and

3) Interdisciplinary capstone projects can be more easily facilitated.

The committee felt that these opportunities were significant enough to proceed with the development of a set of common rubrics.

\subsection{Challenges}

The committee observed four significant challenges to the use of a common set of rubrics for assessing capstone projects. These challenges are the following:

1) Implementations of capstone projects are not uniform across the entire faculty,

2) Rubrics can easily be biased towards a particular discipline,

3) Poor rubrics might lead to an improper assessment of attributes and expectations, and

4) Consistent assessment might not be possible, despite the presence of good rubrics.

The committee felt that none of the challenges were significant enough to abandon the development of a set of common rubrics.

2.2.1. Capstone Project Implementations. At the University of Waterloo, implementations of the capstone project vary from one department to the next. In some departments, students are required to assemble and demonstrate a prototype design. In other departments, students simply need to perform the engineering analysis required to establish the feasibility of a design. Most departments have students work in teams but a few departments allow individual capstone projects. Finally, the number of courses and the weight of the courses varies by department. The various implementations are summarized in Table 1.

Many of the capstone project implementations are similar. Changes to the curriculum are currently in progress to address some of the differences. Some of the proposed changes are a direct result of the committee's work and some of the proposed changes are simply the result of program evolution. Ultimately, it is hoped that all disciplines will eventually converge towards a single implementation of capstone projects. In the interim, the differences were not significant enough to prevent the design of common rubrics.

2.2.2. Rubric Bias. Rubric bias was a significant concern during the design of the common set of rubrics. As an example, a rubric to assess project management skills might look substantially different if developed by a management engineer than a computer engineer. However, the goal was to establish a rubric design that would assess all engineering disciplines in a fair and equitable manner.

To avoid rubric bias, committee members worked in pairs to develop each of the rubrics. The resulting rubrics were then presented to all committee members for comments. Using a process similar to the engineering design process, the rubrics were refined over several iterations of development. Through the use of teams and the use of an iterative design process, bias was effectively reduced.

2.2.3. Rubric Effectiveness. Rubric effectiveness was also a significant concern. The committee debated whether it was possible to develop a rubric that would adequately assess all outcomes without being too generic. In some cases, outcomes simply weren't easily applicable to all disciplines. As such, a compromise was necessary.

To ensure rubric effectiveness, the committee selected a subset of all outcomes to examine. The goal was to select the outcomes that all disciplines could effectively assess. Once a suitable subset of all outcomes was chosen, it was possible to develop a set of common rubrics that each member of the committee agreed would be effective, if used correctly. 
Table 1. Summary of Waterloo Engineering Capstone Project Implementations

\begin{tabular}{||l|c|c|c|c|c||}
\hline \hline \multicolumn{1}{|c|}{ Department } & Compulsory & Courses & Credit Weight & Group Size & Prototype \\
\hline \hline Civil Engineering & Yes & 2 & 1 & 4 & No \\
\hline Chemical Engineering & Yes & 2 & 1 & 2 to 4 & No \\
\hline Computer Engineering & Yes & $3(2)$ & $1.25(1)$ & 4 or 5 & Yes \\
\hline Electrical Engineering & Yes & $3(2)$ & $1.25(1)$ & 4 or 5 & Yes \\
\hline Environmental Engineering & Yes & 2 & 1 & 4 & No \\
\hline Geological Engineering & Yes & 2 & 1 & 1 & No \\
\hline Management Engineering & Yes & 2 & 1 & 3 or 4 & No \\
\hline Mechanical Engineering & No (Yes) & 2 & 1 & 1 to 4 & No \\
\hline Mechatronics Engineering & Yes & 2 & 1 & 1 to 4 & Yes \\
\hline Nanotechnology Engineering & Yes & 3 & 1.25 & 4 & Yes \\
\hline Software Engineering & Yes & 3 & 1 & 4 & Yes \\
\hline Systems Design Engineering & Yes & 3 & 1.5 & 2 to 4 & Yes \\
\hline \hline
\end{tabular}

Items in parentheses indicate changes currently in progress.

2.2.4. Consistent Assessment. Consistent assessment is a requirement if the outcomes assessments from different departments are to be compared effectively. The committee discussed various mechanisms for ensuring the consistent assessment of capstone projects using a common set of rubrics. The discussion was productive as it helped each member of the committee understand how other departments assess student work. As a result, the discussion itself served as a mechanism for promoting consistency.

The committee agreed that consistent assessment was a concern that would not be solved by a simple solution. It was generally agreed that comparisons, even in the presence of inconsistent assessments, would be possible but that such comparisons should only be used to establish general trends.

\section{ATTRIBUTE SELECTION}

As previously indicated, only a subset of the CEAB attributes were selected for assessment as part of capstone project evaluation. There were two reasons for this decision. First, not all attributes are equally applicable to all engineering disciplines. Second, some attributes are better assessed through other mechanisms.

The CEAB attributes chosen for assessment as part of capstone project evaluation were the following:

1) Problem analysis,

2) Design,

3) Individual and team work,

4) Communication skills, and

5) Economics and project management.

Economics and project management was determined to be an outcome with a large scope so this outcome was split into two outcomes. The first one examined engineering economics while the second one examined project management. In total, six rubrics were proposed to assess the five CEAB attributes selected.

\section{ASSESSMENT LEVEL SELECTION}

Selecting the most appropriate set of assessment levels for capstone projects proved to be difficult. The committee investigated examples of publically available capstone project rubrics from several other universities. The number of assessment levels varied from a low of two (unsatisfactory and satisfactory) to a high of seven (ranging from unsatisfactory to outstanding). It was noted that as the number of assessment levels increase, the difficulty of explaining the subtle differences between assessment levels increases.

After much debate over the pros and cons of different assessment schemes, a set of five assessment levels (outstanding, excellent, good, satisfactory, and unsatisfactory) was selected. A level of satisfactory represents the minimum level expected of a graduating student and a level of good represents the minimum level expected of a practicing engineer in the field.

\section{RUBRIC DESIGN}

Six rubrics were developed to assess the five CEAB attributes selected. Due to space limitations, only one of the rubrics is presented in this paper in its entirety. A brief summary of the other rubrics, including a list 
of all the elements is presented for completeness.

\subsection{Communication Rubric}

As an example of one of the rubrics, the communication rubric is shown in Table 2. This rubric can be applied to any course deliverable that demonstrates the use of communication skills. This rubric illustrates the use of the five different assessment levels for each of the selected elements. It should be noted that course instructors may opt out of the evaluation of any element that is not required for a particular deliverable. For example, oral performance would not be an element applicable to a written technical report.

\subsection{Design Rubric}

The design rubric examined the following elements:

- Complexity of the design problem,

- Use of the engineering design process,

- Use of design tools and design techniques,

- Quality of design solution,

- Health and safety considerations,

- Economic considerations,

- Environmental considerations,

- Cultural considerations, and

- Societal considerations.

These elements were chosen after a careful review of the description of the CEAB design attribute. Each element is specifically discussed as a requirement within the context of the CEAB design attribute.

\subsection{Economics Rubric}

The economics rubric was the simplest of the six rubrics. This rubric only examined two elements:

- Feasibility analysis, and

- Economic benefit (e.g., return period assessment, capital cost, etc.).

\subsection{Problem Analysis Rubric}

The problem analysis rubric examined the following five elements:

- Background and context,

- Identification of problems and formulation,

- Suitability of technical analysis,

- Depth of analysis, and

- Conclusions.

\subsection{Project Management Rubric}

The project management rubric examined the following six elements:

- Goals identification,

- Task breakdown,

- Schedule,

- Resources (e.g., human, material, financial, etc.),

- Budget, and

- Risks management.

\subsection{Teamwork Rubric}

The teamwork rubric examined the following five elements:

- Individual contribution to the team,

- Problem solving,

- Collaboration,

- Focus and punctuality, and

- Communication.

It should be noted that the teamwork rubric is not sufficient for a complete assessment of the CEAB attribute as individual work is not assessed. An assessment of individual work can occur in many other settings so it was not a significant rubric design flaw.

\section{OBSERVATIONS}

The design project rubrics were first applied to the Electrical and Computer Engineering design project courses in Winter 2012 with some success. However, a few problems were observed. The problems were not related to the design of the rubrics but rather the problems were related to the way in which the rubrics were presented to the students.

One of the problems encountered was that the rubrics did not align well with the course deliverables. For example, the communication rubric was applied to the final project report in the course. The course instructor presented the rubric in its entirety so some students were confused by the existence of an assessment of oral performance for a written report. This led to some confusion, despite the fact that it was clearly impossible to assess oral communication skills based on a written report. If the course instructor had been involved in the design of the rubric, it is quite possible that the rubric could have been presented better to the students.

Another problem was that the rubrics did not provide the specific details that students wished to know. Many students seem to prefer very specific rubrics as such rubrics can provide great insight into the requirements 
Proc. 2012 Canadian Engineering Education Association (CEEA12) Conf.

Table 2. Faculty of Engineering Communication Rubric

\begin{tabular}{|c|c|c|c|c|c|}
\hline 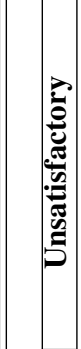 & 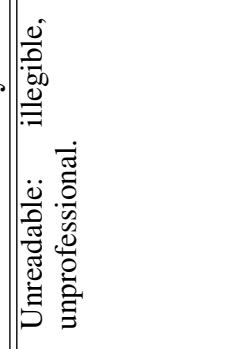 & 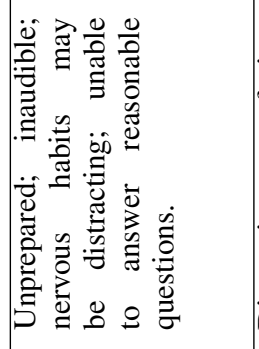 &  & 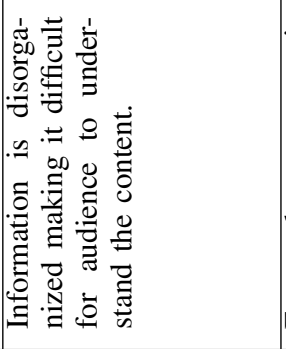 & 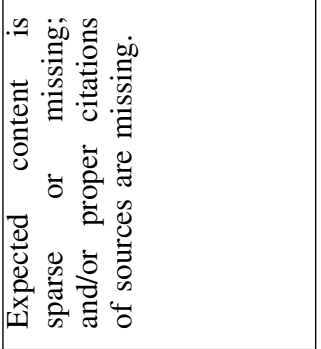 \\
\hline 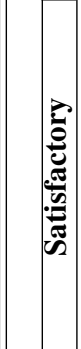 & 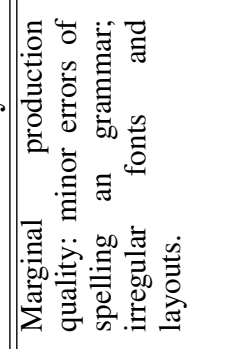 &  & 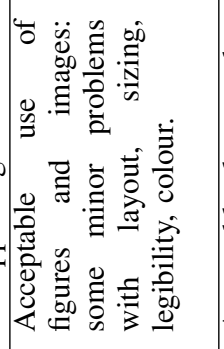 & 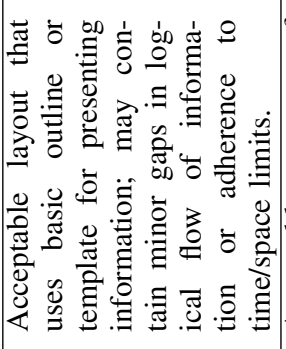 &  \\
\hline 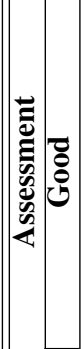 &  &  & 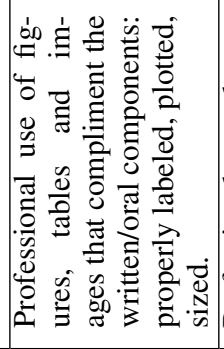 & 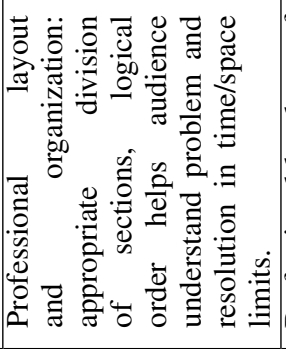 & 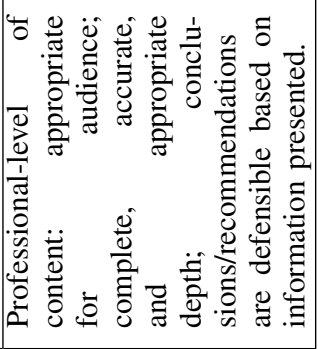 \\
\hline 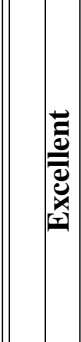 & 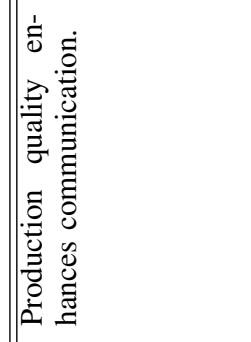 & 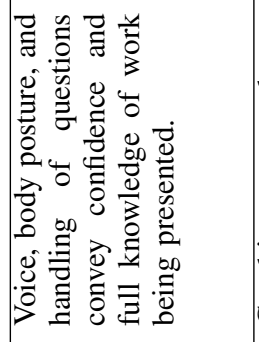 & 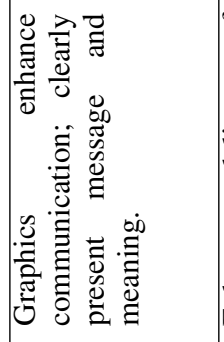 & 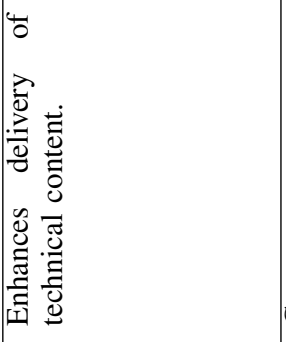 &  \\
\hline 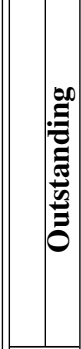 & 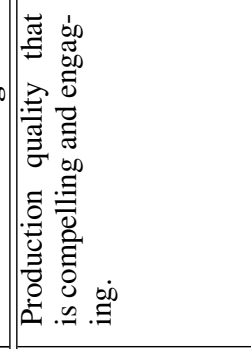 &  &  &  & 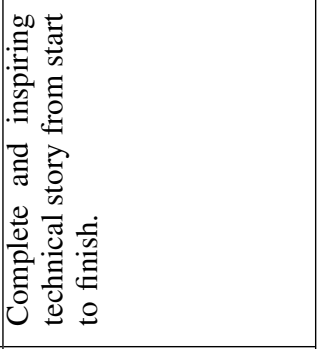 \\
\hline 㦴 &  &  &  &  & $\mid$ \\
\hline
\end{tabular}

CEEA12; Paper 14

Winnipeg, MB; June 17-20, 2012

-6 of $7-$ 
of deliverables and the expectations of instructors. This problem can be resolved by providing more complete descriptions of deliverables and by ensuring that students have a clear notion of the expectations of instructors.

Based on our experiences, we can make the following two observations with respect to capstone project rubric design:

1) Course instructors should be directly involved in the design of the rubrics, and

2) Rubrics should reflect the expectations of deliverables as closely as possible.

\section{CONCLUSIONS}

The challenge of designing rubrics to assess capstone projects in all engineering disciplines is a difficult one. Based on our experience at the University of Waterloo, it is clear that rubrics, much like programs, require continual refinement. However, the goal of creating a common set of rubrics for outcomes assessment in engineering capstone projects is a realistic goal. Not only does rubric creation assist with the assessment of outcomes, rubric creation also encourages reflection upon the curriculum in a way that leads to positive enhancements.

\section{Acknowledgements}

The authors would like to thank Charlie Clarke, Carolyn MacGregor, Dale Henneke, Fathy Ismail, and Ada Zacaj Hurst for their active participation on the committee that developed the rubrics.

\section{References}

[1] L. R. Lattuca, P. T. Terenzini, and J. F. Volkwein. (2006) Engineering Change: A Study of the Impact of EC2000 - Executive Summary. Center for the Study of Higher Education, The Pennsylvania State University. [Online]. Available: http://www.abet.org/uploadedFiles/Publications/Special_ Reports/EngineeringChange-executive-summary.pdf

[2] CEAB. (2011) Accreditation Criteria and Procedures 2011. Canadian Engineering Accreditation Board. [Online]. Available: http://www.engineerscanada.ca/ files/w_Accreditation_Criteria_Procedures_2011.pdf

[3] (2012) Program Review and Accreditation - Background. Centre for Teaching Excellence, University of Waterloo. [Online]. Available: http://cte.uwaterloo.ca/teaching_ resources/OCAV/index.html 